\title{
Lung Transplantation for Adult Respiratory Distress Syndrome after SARS-CoV-2 Infection
}

\author{
Achim Koch ${ }^{10}$ Nikolaus Pizanis ${ }^{1}$ Vasiliki Bessa ${ }^{2}$ Frank Herbstreit ${ }^{3}$ Erich Gulbins ${ }^{4}$ \\ Clemens Aigner ${ }^{5}$ Markus Kamler ${ }^{6,7}$
}

${ }^{1}$ Department of Thoracic and Cardiovascular Surgery, University of Duisburg-Essen Faculty of Medicine, Essen, Northrhine-Westfalia, Germany

2 Department of Pulmonology, University of Duisburg-Essen Faculty of Medicine, Essen, Northrhine-Westfalia, Germany

${ }^{3}$ Department of Anesthesiology, University of Duisburg-Essen Faculty of Medicine, Essen, Northrhine-Westfalia, Germany

${ }^{4}$ Institute for Molecular Biology, University of Duisburg-Essen Faculty of Medicine, Essen, Northrhine-Westfalia, Germany

${ }^{5}$ Department of Thoracic Surgery, Universitat Duisburg-Essen

Medizinische Fakultat, Essen, Germany

${ }^{6}$ Thorax und Kardiovaskuläre Chirurgie, Universitätsklinikum Essen,

Essen, Germany

${ }^{7}$ Herzchirurgie, Herzzentrum Essen, Essen, Germany

Thorac Cardiovasc Surg Rep 2022;11:e23-e26.

\begin{abstract}
Address for correspondence Prof. Dr. med. Achim Koch, Department of Thoracic and Cardiovascular Surgery, University of Duisburg-Essen Faculty of Medicine, Hufelanstrasse 55, Essen, Northrhine-Westfalia 45122, Germany (e-mail: achim.koch@uk-essen.de).
\end{abstract}

\begin{abstract}
Keywords

- SARS-CoV-2

- lung transplantation

- sphingosine

Background The majority of patients with severe acute respiratory syndrome coronavirus-2 (SARS-CoV-2) infection present mild symptoms. However, some patients develop severe acute respiratory distress syndrome (ARDS) and subsequent irreversible lung damage despite extracorporeal membrane oxygenation, leaving lung transplantation the ultimate therapeutically option.

Case Description Here, we report a case of lung transplantation in a 31-year-old male recipient suffering from post-coronavirus disease 2019 respiratory failure with irreversible ARDS after prolonged extracorporeal membrane oxygenation therapy.

Conclusion Patient selection criteria are elucidated. One relevant mechanism for susceptibility to SARS-CoV-2 in the respiratory system, the acid sphingomyelinase/ ceramide system might be altered during infection with SARS-CoV-2.
\end{abstract}

\section{Case}

We report on a case of lung transplantation in a 31-yearold male recipient suffering from post-coronavirus disease 2019 (COVID-19) respiratory failure with irreversible acute respiratory distress syndrome (ARDS) after prolonged extracorporeal membrane oxygenation (ECMO) therapy.

received

August 31, 2021

accepted after revision

November 10, 2021
DOI https://doi.org/ $10.1055 / \mathrm{s}-0042-1742714$. ISSN 2194-7635.
The patient was admitted to hospital with fever, dyspnea and polymerase chain reaction (PCR) testing confirmed severe acute respiratory syndrome coronavirus-2 (SARSCoV-2) infection on November 9, 2020. Within 2 days, mechanical ventilation became mandatory. On the 8th day, a tracheostomy was performed. After temporary improvement under maximized therapy with steroids, antibiotics, and antifungal therapy, 21 days later venovenous ECMO was

(c) 2022. The Author(s).

This is an open access article published by Thieme under the terms of the Creative Commons Attribution-NonDerivative-NonCommercial-License, permitting copying and reproduction so long as the original work is given appropriate credit. Contents may not be used for commercial purposes, or adapted, remixed, transformed or built upon. (https://creativecommons.org/ licenses/by-nc-nd/4.0/)

Georg Thieme Verlag KG, Rüdigerstraße 14, 70469 Stuttgart, Germany 
necessary, temporary hemofiltration was required for 2 days, and heparin-induced thrombocytopenia type II was diagnosed.

After failure of repeated weaning attempts from ECMO, the patient was referred for the evaluation of lung transplantation on day 56 after hospital admission. The decision to accept the patient on the waiting list took the following considerations into account: (1) no potential for recovery, several unsuccessful weaning attempts; (2) mono-organ failure; (3) three repetitive viral cultures taken by nasopharyngeal swabs as well as bronchoalveolar lavage were negative; (4) patient mobilized on ECMO, deconditioning was not too advanced; (5) patient was awake, able to give full consent; (6) Crohn's disease diagnosed 2 years ago was well controlled with low-dose immunosuppression and under treatment in our center. No other comorbidities were present; (7) normal body mass index of 28; (8) no alternative treatment options; (9) a supportive family.

\section{Surgery and Course}

The patient was transplanted following our institution standard protocol with donor lungs from a 28 years old male donor with intracerebral hemorrhage as cause of death. ECMO was intraoperatively switched to venoarterial mode. To achieve adequate donor-recipient size match, a lung volume reduction (middle lobe and lingula resection) was necessary. Ischemic time for right lung was 332 minutes and left lung was 480 minutes. Postoperative pulmonary gas exchange was excellent P/F 348 at 1.0 and ECMO was discontinued. Primary graft dysfunction at 24,48 , and 72 hours was 0 according to
International Society of Heart and Lung Transplantation (ISHLT) criteria. Immunosuppression was applied according to our institutional standard protocol (antithymocyte globulin, tacrolimus, mycophenolate mofetil, and steroids). A superinfection with Serratia marcescens was found in culture of the recipient lung as well as Candida glabrata in the pleural culture that was treated by antibiotics and antimycotic treatment with voriconazole. Since Mycobacterium tuberculosis DNA was found in the donor lung culture, a prophylaxis with isoniazid was initiated. The initial postoperative course was uneventful with a prolonged weaning phase until day 36 . A gastroparesis necessitated temporary placement of a jejunal feeding tube. The patient was further mobilized, and underwent sternal revision for instability without signs of infection on postoperative day 59 . He was discharged to rehabilitation on postoperative day 86 (-Fig. 1).

\section{Recipient Pathology}

Recipient lungs were edematous with consolidated, brownish tissue and pleural fibrous adhesions. The lung parenchyma showed large areas of atelectasis with small parts of remaining alveoli, interstitial expansion by fibrosis, and areas of organized pneumonia. SARS-CoV-2 nucleoprotein or microthrombi were not found. The pattern was classified as a nonspecific interstitial pneumonia with a diffuse alveolar damage, matching post-COVID-19 pneumonia.

\section{Focus on Sphingolipids}

Staining histology sections with antibodies against ceramide, acid ceramidase, sphingosine, and sphingosine kinase
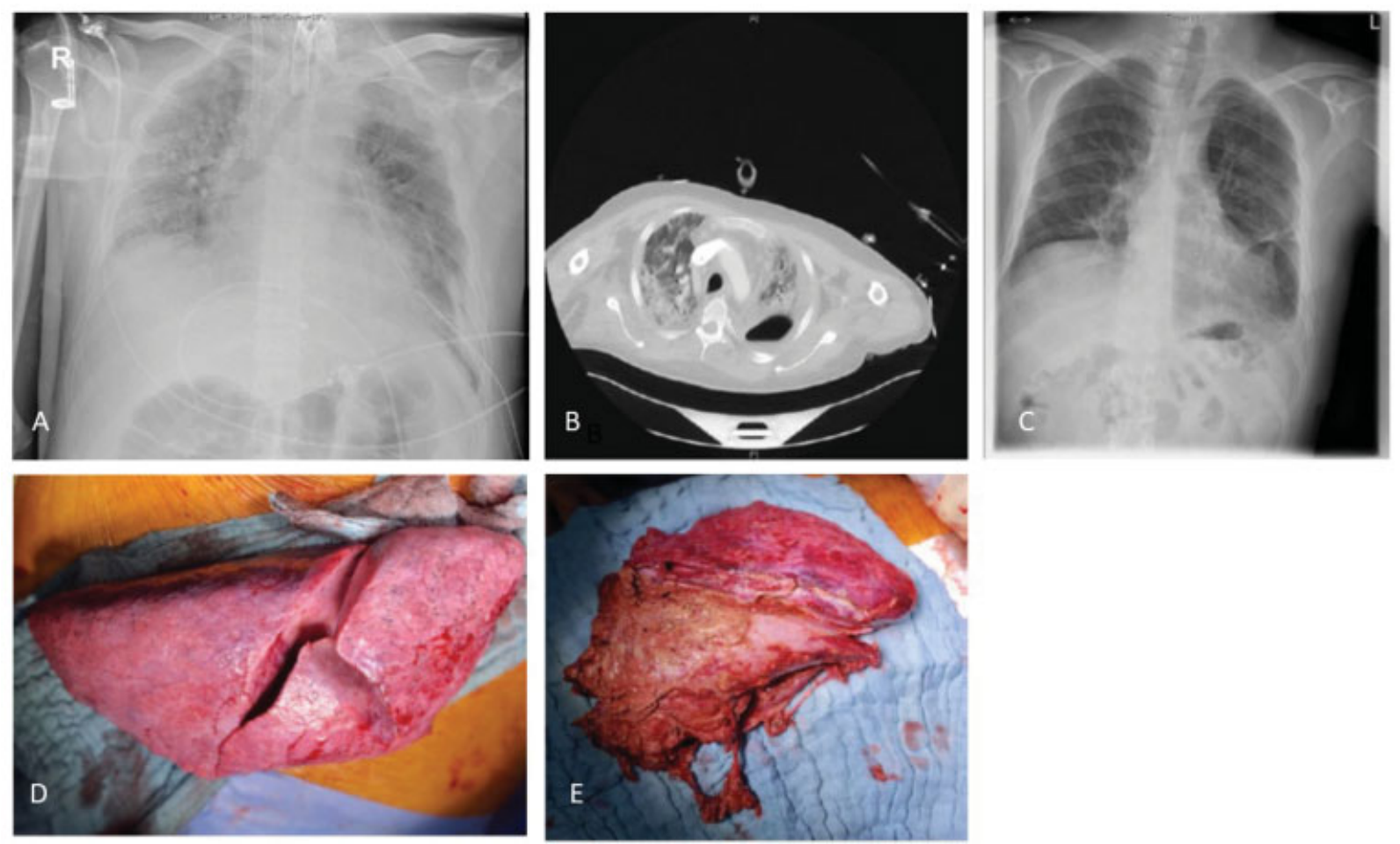

Fig. 1 Chest X-ray (A) and computed tomographic scan (B) on venovenous extracorporeal membrane oxygenation before lung transplantation. Chest X-ray before discharge (C), intraoperative view of the right recipient lung; macroscopic view has gross appearance as idiopathic pulmonary fibrosis (D), macroscopic view of the left recipient lung. Formation of large abscess with fibrinous tissue (E). 

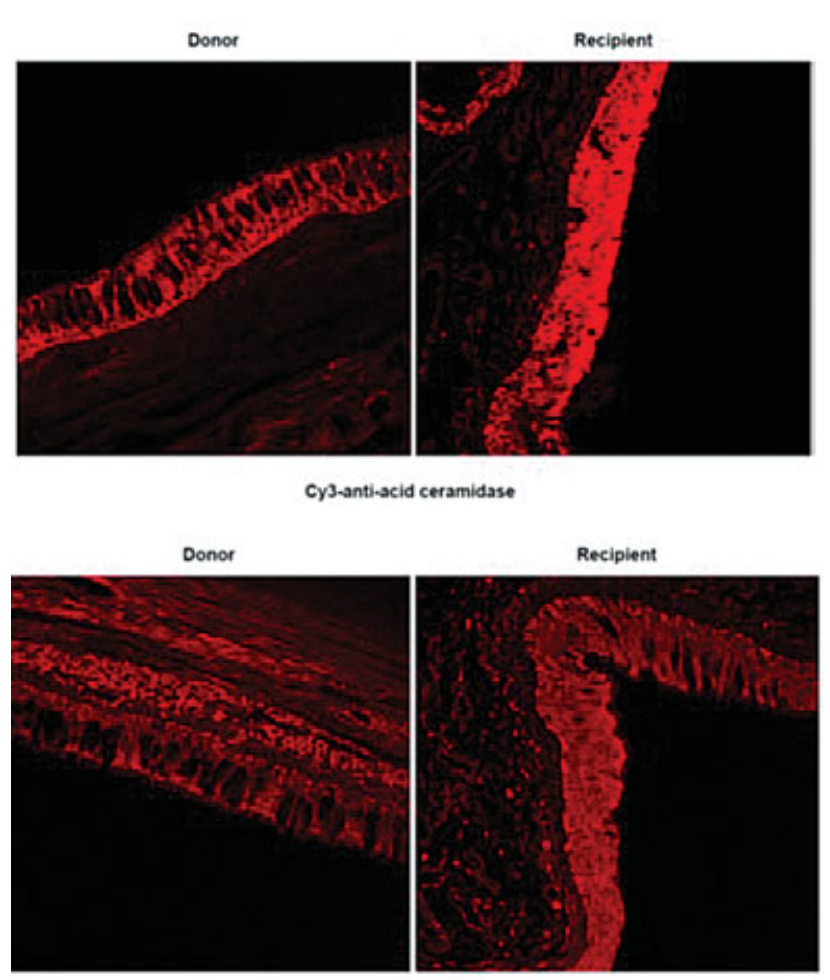

Cy3-anti-sphingosine

Fig. 2 Sphingolipid metabolism is markedly altered in recipient epithelial cells. Sections from human recipient or donor lungs were stained with Cy3-coupled antibodies against acid ceramidase $(1: 1000$, ProSci) and sphingosine (1:1000, Alfresa Pharma Corp.) as previously described. ${ }^{9}$ Representative results from the analysis of at least 20 different areas of the lung from the donor and the recipient are shown in the figure.

2 revealed a marked increase in the expression of acid ceramidase and of sphingosine in the epithelial cell layer in the recipient (-Fig. $\mathbf{2}$ ).

\section{Discussion}

We report on a successful lung transplantation in a patient with post SARS-CoV-2-associated ARDS. We found differences in the ceramide/sphingosine metabolism, suggesting postinflammatory changes in the recipient lung.

Lung transplantations due to SARS-CoV-2 infection have been reported only from few centers. ${ }^{1-3}$ Even though some recommendations were made based on expert opinion, decision making for this indication is still undefined and potential outcome unclear. ${ }^{4,5}$ Our center's policy mandates that all patients have to have equal opportunity for transplantation in regard to medical indications or contraindications and the resulting expected survival benefit. ${ }^{6}$ Routine workup follows a standardized protocol and, in this case, the decision was based on young age, single-organ dysfunction of the lung, irreversible lung injury, and established fibrosis, patient ability to consent to transplantation and to participate in physical rehabilitation, negative SARS-CoV-2 PCR test results, and body mass index normal. Patient was ambulatory for well-controlled Crohn's disease.
However, Crohn's disease might be a cofactor for the development of COVID-19 infection in this patient. ${ }^{7}$

We investigated whether other confounders such as potentially compromised epithelial cells from the recipient exhibit an altered immune/sphingolipid/ceramide metabolism. It has been shown by our group and others that the sphingolipid ceramide is central for inducing lung fibrosis and promotion of chronic bacterial pneumonia, for instance, in cystic fibrosis and some viral infections. ${ }^{8,9}$ In contrast, sphingosine protects from bacterial pneumonia ${ }^{9,10}$ and even some viral infections. ${ }^{10}$ Finally, we have shown that the acid sphingomyelinase/ceramide system is required for infection of cells with SARS-CoV-2, which is blocked by sphingosine. $^{8,10}$ Therefore, we investigated whether the epithelial cells from the recipient exhibit an altered sphingolipid metabolism.

We detected a marked increase in the expression of the acid ceramidase and of sphingosine in the epithelial cell layer. Sphingosine directly kills bacterial pathogens ${ }^{9}$ and therefore we interpret the increase in acid ceramidase and sphingosine as a chronic antimicrobial mechanism upregulated in the recipient epithelial cell layer.

\section{Conclusion}

We report on a 31 years old patient with post-SARS-CoV2-pneumonia, 8 weeks course on ECMO due to irreversible pulmonary destruction, and successful lung transplantation. In selected patients with SARS-CoV-2-related irreversible pulmonary destruction, lung transplantation may be a feasible treatment option. Investigation of metabolic processes shall increase the knowledge about susceptibility, prognostic value, and potential therapeutic consequences.

\section{Authors' Contributions}

Achim Koch was involved in conception of design, data acquisition, interpretation, drafting, and final approval and was accountable for all aspects of the work, and was also a corresponding author.

Nikolaus Pizanis was involved in conception of design, data acquisition, interpretation, drafting, and final approval and was accountable for all aspects of the work.

Vasiliki Bessa was involved in acquisition of data and revision and final approval and was accountable for aspects of accuracy and integrity and revision.

Frank Herbstreit was involved in acquisition of data and revision and final approval and was accountable for aspects of accuracy and integrity.

Erich Gulbins was involved in design of work and revision and final approval and was accountable for aspects of accuracy and integrity.

Clemens Aigner was involved in interpretation of data and revision and final approval and was accountable for aspects of accuracy and integrity.

Markus Kamler was involved in conception and critical revision and final approval and was accountable for aspects of accuracy and integrity. 
e26 Lung Transplantation for ARDS after SARS-CoV-2 Infection Koch et al.

\section{Conflict of Interest}

None.

\section{References}

1 Chen JY, Qiao K, Liu F, et al. Lung transplantation as therapeutic option in acute respiratory distress syndrome for coronavirus disease 2019-related pulmonary fibrosis. Chin Med J (Engl) 2020; 133(12):1390-1396

2 Han W, Zhu M, Chen J, et al. Lung transplantation for elderly patients with end-stage COVID-19 pneumonia. Ann Surg 2020; 272(01):e33-e34

3 Lang C, Jaksch P, Hoda MA, et al. Lung transplantation for COVID19-associated acute respiratory distress syndrome in a PCRpositive patient. Lancet Respir Med 2020;8(10):1057-1060

4 Cypel M, Keshavjee S. When to consider lung transplantation for COVID-19. Lancet Respir Med 2020;8(10):944-946

5 Bharat A, Querrey M, Markov NS, et al. Lung transplantation for patients with severe COVID-19. Sci Transl Med 2020;12(574):4282
6 Bharat A, Machuca TN, Querrey M, et al. Early outcomes after lung transplantation for severe COVID-19: a series of the first consecutive cases from four countries. Lancet Respir Med 2021;9(05): 487-497

7 Brenner EJ, Ungaro RC, Gearry RB, et al. Corticosteroids, but not TNF antagonists, are associated with adverse Covid-19 outcome in patients with inflammatory bowel diseases: results from an international registry. Gastroenterology 2020;159(02): 481-491.e3

8 Carpinteiro A, Edwards MJ, Hoffmann M, et al. Pharmacological inhibition of acid sphingomyelinase prevents uptake of SARSCoV-2 by epithelial cells. Cell Rep Med 2020;1(08):100142

9 Grassmé H, Henry B, Ziobro R, et al. $\beta 1$-Integrin accumulates in cystic fibrosis luminal airway epithelial membranes and decreases sphingosine, promoting bacterial infections. Cell Host Microbe 2017;21(06):707-718.e8

10 Edwards MJ, Becker KA, Gripp B, et al. Sphingosine prevents binding of SARS-CoV-2 spike to its cellular receptor ACE2. J Biol Chem 2020;295(45):15174-15182 